\title{
Real Time Net Zero Energy Building Energy Manager with Heterogeneous Wireless Ad hoc Network Adaptable To IoT Architectures
}

\author{
Raja Vara Prasad $Y^{*}$, Shubham Goel ${ }^{* *}$, Rajalakshmi P* and U. B. Desai* \\ *Department of Electrical Engineering, IIT Hyderabad, India, Email: [ee10p004 raji ubdesai]@iith.ac.in \\ ** Department of Electronics Engineering, NIT Uttarakhand, India, shubham11goel@ gmail.com
}

\begin{abstract}
Significant energy consumption by buildings from utility grid has made researchers revisit existing Building Energy Management Systems (BEMS). Most of the developing countries have taken a green initiative of Net Zero Energy Buildings (NZEB) to reduce carbon foot print and fast depletion of conventional energy sources. Though the integration of solar and wind based systems to grid is successful in recent years, residential building energy management systems with renewable energy sources is still an evolving research area. Monitoring, control and actuation systems should be tightly coupled with the help of any to any device communication namely Internet of Things (IoT) to realize an efficient NZEB. In this paper a real time NZEB is proposed and developed with bidirectional wireless sensor and actuation system. Proposed NZEB central server collects and maintains a database of on site solar generation, battery state of charge and load power consumption data of a building with help of IEEE 802.15.4 and IEEE 802.11 wireless networks. Proposed system was deployed as a test bed with sensing, control, actuation and server modules and connecting them with a bidirectional wireless network architecture similar to IoT. Data observed at experimental test bed confirm that developed system can estimate on site solar power generation, state of charge on battery bank and load power consumption with negligible error. A simulation study with experimental data collected at NZEB test bed shows that NZEB can optimally schedule loads between local generation and utility grid thereby minimizing peak demand on the grid.
\end{abstract}

Keywords-State of Charge, Net Zero Energy Building, IoT, Solar radiation, Wireless Ad hoc networks.

\section{INTRODUCTION}

According to recent studies in [1] and Smart-2020 report in [2], energy consumption of buildings are $40 \%$ of total energy consumption resulting in large scale of green house gases. Past, present and the future predictions of energy usage and available conventional energy reserves are presented in [1]. Study in [1] also stresses on need of converting present buildings into green buildings or Net Zero Energy Buildings (NZEB). In order to reduce green house gases, there may be an immediate urgency to integrate renewable source of energy such as solar energy with every residential building to meet our increase in energy requirement. Solar energy is becoming one of the significant energy sources in the world's energy proficiency. According to [3], India has a high insolation (measure of solar radiation energy received on a given surface area in a given time) which makes solar power a better option in India. According to [4], there are several steps taken by U.S to become less dependent on conventional sources of energy and move to renewable sources which reduces 60 to 70 percent of conventional sources of energy in buildings. Buildings with such efficient energy management system and renewable energy sources that can generate an equal energy to the amount they consume in a year are named as Net zero Energy Buildings.

A layer wise theoretical architecture of Internet of Building Energy Systems is discussed in [6]. Even though architecture is proposed for complete NZEB, the practical implementation difficulties are not answered in [6]. A Multi Agent System (MAS) based Building energy manager is proposed and well investigated in [7] for different aspects like cooling, heating and power. Agent models in [7] could not capture the communication aspects of the ZEB. A Cyber Physical System model to realize a NZEB is claimed in [8]. Even though authors proposed a $3 \mathrm{D}$ reconstructive model to identify loads and to place sensors, model in [8] lacks efficient communication architecture and experimental validation. An effective Graphical interfaced based ZEB is well illustrated in [9]. Study in [9] is limited to effective visualization of energy, water and other essential resources in the building, but could not give a complete solution for an efficient NZEB.

Most of the NZEB models in the literature which can import/export power to the grid are using either wired communication or vendor specific proprietary modules which cannot be extended to existing buildings due to major rewiring or higher investments. Hence there is a higher need for general architecture of building energy management system which has information of local energy sources and load consumption. This paper provides a novel architecture of building energy management system to convert existing buildings into net zero buildings using real time load, on site solar generation and battery charge data at the central server using multi gateway IoT ready wireless communication as back bone. Proposed system depends on inputs like load monitoring data using power monitoring module, solar radiation data from sensor and state of charge estimation of battery bank. All these data are forwarded to central server using IEEE 802.15.4 and IEEE 802.11 wireless devices. Real time data at central server can improve decisions of the proposed NZEB, thereby selecting an 


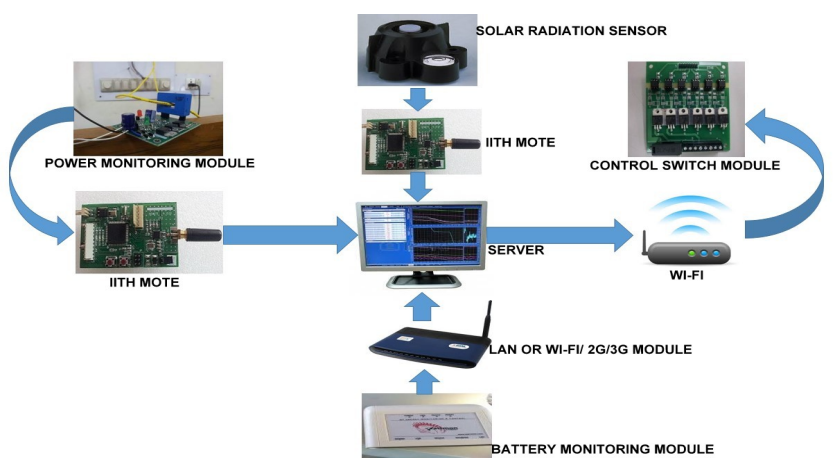

Fig. 1: Block diagram of the proposed system

optimal energy source from existing options. Remainder of the paper is organized as follows. Section-II gives description of proposed system architecture. Section-III briefs experimental set up and observations along with simulation results. SectionIV concludes with future scope of work.

\section{System ARCHITECTURE}

System architecture proposed for solar based NZEB in this paper is shown in the Fig. 1. Proposed NZEB is a layered architecture, where each layer is a functional block of total architecture. Different layers in the proposed model are Sense, Communication, Control/Actuation and Server. Power monitoring, solar radiation and battery status module are part of sense layer. IEEE 802.15.4 and IEEE 802.11 based Wireless communication modules are part of communication layer. Embedded controller with power electronic modules functions as control and actuation layer. A Java based decision engine adaptable to intelligent actions is the server which is connected to multiple communication gateways. Detailed description of individual modules in the system architecture is given in following subsections.

\section{A. Sense Layer}

Sense layer of the proposed system is realized by using solar irradiance sensor, power monitoring and state of charge modules. A brief description of each module is given below.

1) Solar Radiation Sensor: Davis solar irradiance sensor [11] is used to measure global radiation at the given location shown in the Fig. 2. Solar radiation is measured in real time and stored into database to empower server with statistical data of solar insolation for optimal decision and control. Analog voltage generated from the sensor is conditioned and given as an input to IEEE 802.15.4 compliant IITH mote developed by IIT Hyderabad [10]. Analog voltage of solar irradiance sensor is converted into meaningful watt/sqm (sqm means square metre) using equation (1) in data sheet of [11].

$$
I_{g}=\frac{A D C_{\text {out }} * V_{\text {ref }}}{k * A D C_{\max }}
$$

where $I_{g}$ is global solar irradiation value in watt/squaremetre, $A D C_{\text {out }}$ is present equivalent $\mathrm{ADC}$ value of sensor, $A D C_{\max }$ is maximum value of $\mathrm{ADC}, \mathrm{K}$ is sensor constant which is $1.67 \mathrm{millivolt} /\left(\right.$ watt/squaremetre) given in [11] and $V_{\text {ref }}$ is

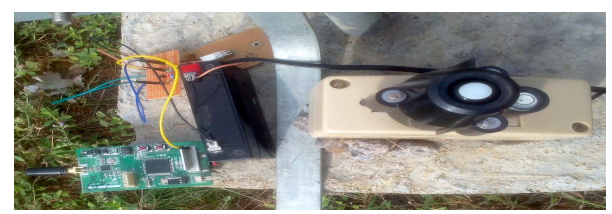

Fig. 2: Solar radiation sensor at IIT Hyderabad test bed

maximum output voltage of the sensor i.e 3 volts. From available global radiation $I_{g}$ at a given location, only part of the radiation can be converted into useful electrical power by photo voltaic sells as shown in the equation (2). There are many factors which contribute to these losses, mainly inverter, temperature, cable, dust, shadowing and inclination. These losses are accounted in PR factor in the equation (2). IITH mote is programmed to collect and send data to central server either in single hop/ multi-hop fashion depending on distance from central server. IITH gateway module connected through serial port to the server receives the data and organizes into the database of the server. Data collected at IITH smart lab is as shown in the Fig.3. Fig.3 plots global radiation $I_{g}$ in watt/sq.m versus time in hours. $I_{g}$ is found increasing with increase from $10 \mathrm{AM}$ to $1 \mathrm{PM}$ after which it is decreasing. This pattern of increase in $I_{g}$ is due to general tendency of increase in solar power from morning to afternoon after which it gradually decreases. Sudden fluctuations in $I_{g}$ are observed at 1:30 PM and 15:30 PM due to clouds.

$$
E_{\text {est }}=A * \eta * I * P R
$$

where $E_{\text {est }}$ is the solar generated energy by PV panel in Kwhr, A is the area of solar panel, $I_{g}$ is global solar insolation, $\eta$ is the panel efficiency/ yield factor and PR is the performance factor including many losses like inverter, temperature, cable, dust, shadowing and inclination etc.

2) Battery Monitoring Kit: Solar system is generally accompanied by energy storage systems like battery banks to store energy and discharge it when utility grid is either absent or experiencing peak demand. State of charge (SoC) and voltage of battery bank is monitored in real time to power loads from battery bank instead of utility grid when grid has peak demand of load. This module measures the state of charge through coulomb counting method according to [12]. Battery monitoring module namely "wattmon" [12] is used in proposed model shown in Fig. 1. SoC in Ampere hour left in the battery bank can be forwarded to server using wired LAN or wireless $2 \mathrm{G} / 3 \mathrm{G}$ connection. Battery monitoring module used in IITH testbed is of 75 amps current sensor and 300 volts voltage sensor to measure charging and discharging currents and voltages respectively. Maximum SOC capacity of installed system in IIT Hyderabad is $160 \mathrm{Ah}$. Real time data of battery bank SoC can be logged into central server. Please refer to [12] for detailed description of the module.

3) Power Monitoring Module: Real time load consumption of a building is an important input to the server to intelligently switch between solar, available battery and utility grid. Power monitoring module is developed by IIT Hyderabad to monitor 


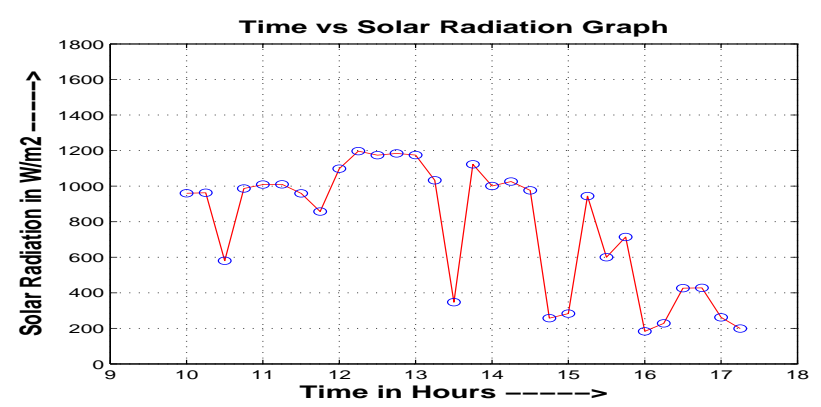

Fig. 3: Time vs Solar radiation graph

dynamic loads in the building such that large dynamic loads status is known to the server in real time. Server can either estimate the load for the next time slot or can take a schedule decision for present time slot depending on the data sent from power monitoring module to server using Wi-Fi communication module. Implementation details can be found in [13] and [14] for better understanding.

\section{B. Control and Actuation Layer}

Control module is proposed in the architecture to switch between different available energy options. Central server runs a decision algorithm after collecting data from different modules. These decisions from server are converted into electrical actuation signals using embedded board and power electronic actuators. Developed control module at IIT Hyderabad can only switch on/off the load but cannot control load in case of variable loads. Adaptive rule engine in the central server with all above inputs is responsible for schedule and control of devices. Automation of electrical devices is not implemented in the initial tet bed. A simulation model is developed instead of full scale experimentation of the proposed system. Finer details of control module can be found at [13] and [14].

\section{Communication Layer}

Wireless communication based novel heterogeneous architecture proposed in this paper to collect and forward the load status, State of charge (SoC) and solar radiation information to the central server. IEEE 802.15.4 and IEEE 802.11 compliant devices are used to connect all devices to form an IoT network shown in the Fig. 1. IITH mote is programmed in TinyOs language to send and receive data from server. RCM 5600 based Wi-Fi modules in [14] are also programmed in Embedded C language to collect SoC data. Wi-Fi modules are also programmed to perform as interfacing embedded board between server and electrical actuation system. Details of the above two modules are in [13] and [14].

\section{Server Layer}

Central server connected to respective gateways like IEEE 802.15.4 and IEEE 802.11 through UART communication. Central server based on Java collects and organize data into database. Battery bank SoC is also logged into central server using LAN connection. A decision engine is developed to best utilize local solar energy first and decide between battery SoC and utility grid according to real time load status when pricing is high. Server conveys control decisions to respective actuation modules to optimize usage of energy thereby converting buildings into NZEB. Present algorithm can be made adaptive to make NZEB as building block to smart grids.

\section{EXPERIMENTAL RESULTS}

Proposed system architecture shown in the Fig. 1 is implemented as a test bed in IIT Hyderabad smart lab to collect experimental data. All modules are installed according to the description in the previous section. A 5 Kilowatt solar Photo voltaic system is installed to charge available battery bank of a 96 Volt 160 Ah Battery bank with 96 volt DC to 240 Volt AC inverter. Solar global radiation sensor is installed near PV panels to measure solar radiation. Generated solar power readings $P_{\text {act }}$ in inverter is also noted down manually at regular intervals of 15 minutes. Solar radiation data with help of IITH mote network is also collected at server. SoC data of battery bank associated with $5 \mathrm{Kw}$ is saved into central server. Real time experimentation of the proposed model was difficult to implement in IITH smart lab because of maximum connected load of $8.6 \mathrm{Kw}$ is much higher than solar capacity of $5 \mathrm{Kw}$ which may create stability problems due to dynamic load variations. Instead of experimentation on test bed a simulation model is created for NZEB to observe the benefits of our proposed real time intelligent load switching for NZEB. Since solar radiation is observed from 10.00 AM to 5.00 PM at the site total simulation results can be observed for the same time frame. Simulation algorithm flow and results are explained in next subsection as follows.

\section{A. Simulation Model}

Experimental data stored in server are used for MATLAB based NZEB model simulation. Simulation is modeled to maximize on site renewable energy consumption and shifts to battery bank and utility grid respectively which is a virtual Net Zero Energy Building. Simulation model observes data from input database and perform decisions accordingly for every 15 minute. Simulation model flow is given in Fig. 4. Algorithm flow starts with collection of available solar generation data, battery SoC and load status for that considered time slot. Algorithm logically tries to allocate present solar generation and if load is still higher, it takes energy from battery bank and utility grid. We assume that solar radiation and load remain constant for 15 minutes of time and load as uniform random variable. We also assumed that maximum capacity of battery bank is $60 \%$ as battery cannot discharge below $40 \%$.

\section{B. Simulation Results}

Simulation model in this paper considered the smart lab loads. We generated load value for simulation module by generating a random vector of loads which decides the real time capacity of smart lab. Dynamic loads in the lab are also modelled using a uniform random function in MATLAB. Real time load value for simulation model is explained with an example as below. There are 10 Constant lighting loads, 5 


\begin{tabular}{|c|c|c|c|c|c|c|c|c|c|c|c|c|c|c|c|c|c|c|c|c|c|}
\hline$L_{R}(n)$ & 1 & 0 & 1 & 1 & 1 & 0 & 0 & 0 & 1 & 0 & 1 & 1 & 0 & 1 & 0 & 0 & 0 & 0 & 1 & 0 & 0 \\
\hline$L_{c}(n)$ & 40 & 40 & 40 & 40 & 40 & 40 & 40 & 40 & 40 & 40 & 80 & 80 & 80 & 80 & 80 & & & & & & \\
\hline
\end{tabular}

TABLE I: Example random schedule vector $\left(L_{R}\right)$ and constant load vector $\left(L_{c}\right)$

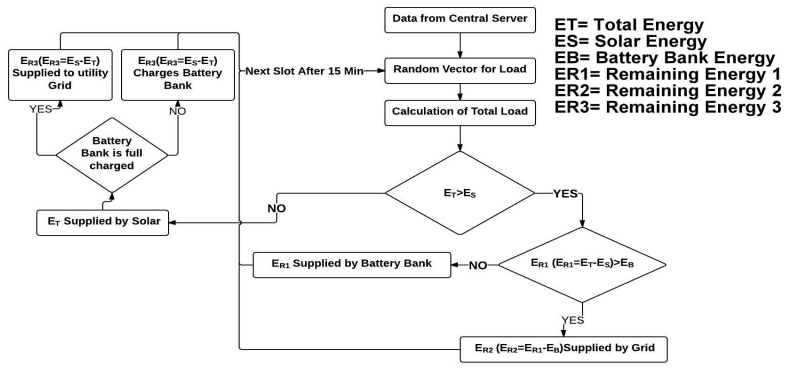

Fig. 4: Simulation Algorithm for the proposed NZEB

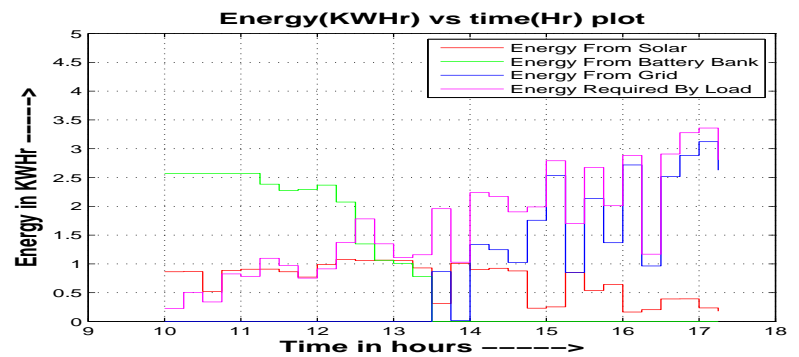

Fig. 5: Simulation result of the proposed algorithm

constant server loads, 3 dynamic air conditioning loads $\left(L_{\min }\right.$ $=1000$ to $L_{\max }=1600$ watts $)$ and 3 dynamic lab power electronics lab equipment loads $\left(L_{\min }=500\right.$ to $L_{\max }=1000$ watts) in IITH testbed. Randomness in switching schedules of these loads are considered by generating a random vector of 21 in size for total 21 loads. Similarly dynamic behaviour of 6 loads is considered by generating a uniform random variable for each load. Total load is calculated as shown in below example. Random schedule vector $\left(L_{R}\right)$, Constant load vector $\left(L_{c}\right)$ and dynamic load vector $\left(L_{D}\right)$ are given in Table. 1. There are 21 load schedules for each time interval of 15 minutes. In vector $L_{R}, 1$ represent load is on and 0 represent load is off. Constant load $\left(L_{C}\right)$ is the product of $L_{R}$ vector and $L_{c}$ vector.

Dynamic load : $\left(L_{D}\right)$ is $0+0+0+1 *\left(L_{\text {min }}+\left(L_{\text {min }}{ }^{-}\right.\right.$ $\left.L_{\max }\right)^{*}$ rn). Finally total load is given in equation (3)

$$
L_{T}=L_{C}+L_{D}
$$

Rules stated in Fig. 4 are simulated to observe proposed real time NZEB benefits. Fig. 5 plots 4 curves i.e load curve $L_{T}$, Solar generation $E_{e s t}$, SoC value of battery bank and finally grid energy. It can be observed from the plot that real time solar generation is sufficient for load system and does not take any power from battery bank or grid during 10 AM to 11 AM. Solar on site generation starts decreasing from 11 AM due to which battery SoC starts decreasing. Battery SoC was almost $40 \%$ at 1:30 PM. We considered a minimum limit of $40 \%$ on battery bank below which it cannot supply to load. Finally after 1:30 total load is taken over by utility grid and instantaneous solar generation. Results show that proposed model is behaving like a real time NZEB maximizing local energy usage and minimizing usage from utility grid energy.

\section{CONCLUSION}

Net Zero Energy buildings (NZEB) system with real time power monitoring, solar radiation and battery state of charge data is realized with well known wireless standards like IEEE 802.15.4 and IEEE 802.11. Experimental data are collected at test bed of IIT Hyderabad smart building. A simulation model is developed to analyse the benefits of proposed NZEB server. Simulation model results try to maximize the usage of local energy like solar and battery SoC thereby reducing peak load on utility grid. Proposed NZEB with adaptive rule engine based control algorithm is the future scope of our work.

\section{REFERENCES}

[1] Satyen Mukherjee,'Opportunities and Challenges with Net Zero Energy Buildings", Proceedings of the 23rd International Symposium on Power Semiconductor Devices and IC's May 23-26, 2011 San Diego, CA

[2] "Smart 2020 report" http://www.smart2020.org/_assets/files/ Smart2020UnitedStatesReportAddendum.pdf

[3] http://en.wikipedia.org/wiki/Solar_power_in_India

[4] Lukas Spitalny, Dennis Unger, Jan Teuwsen, Volker Liebenau, Johanna M.A. Myrzik,Bart Van Reeth,'Effectiveness of a Building Energy Management System for the Integration of net Zero Energy Buildings into the Grid and for Providing Tertiary Control Reserve," PowerTech IEEE Grenoble 2013, pp. 1-6

[5] Thanh-LamNguyen, Ying-Fang Huang,Ming Hung Shu, Bi-Min Hsu, "Forecasting Models For the Solar Photo voltaics consumption in United states of America", Conference on Power and Energy IPEC 2012, pp. 284-288

[6] Chen Yongpan, Zhang Jili, Mu Xianmin, Ma Jinxing, "Study on the theoretical framework of the internet of building energy systems," 5th International Conference on Computer Sciences and Convergence Information Technology (ICCIT), pp.973,976, Nov. 30 2010-Dec. 22010

[7] Peng Zhao, Suryanarayanan, S., Simoes, M.G., "An Energy Management System for Building Structures Using a Multi-Agent Decision-Making Control Methodology," IEEE Transactions on Industry Applications vol.49, no.1, pp.322,330, Jan.-Feb. 2013

[8] Kun Hua, "Intelligent controls for cyber physical energy buildings," IEEE/CIC International Conference on Communications in China - Workshops (CIC/ICCC), pp.120,124, 12-14 Aug. 2013

[9] Bartram, L., Rodgers, J., Muise, K., "Chasing the Negawatt: Visualization for Sustainable Living," IEEE transacctions on Computer Graphics and Applications, vol.30, no.3, pp.8,14, May-June 2010

[10] http://www.iith.ac.in/ raji/downloads/IITH-mote-webpage.pdf

[11] Davis solar radiation sensor datasheet $\mathrm{http}: / / \mathrm{www}$.davisnet.com

[12] Wattmon High voltage DC kit datasheet http://www.wattmon.com

[13] Rajavara Prasad Y, Bharathi, Alok Kumar, P. Rajalakshmi, U B Desai,"WSN based power monitoring in smart grids", IEEE International Conference on Intelligent Sensors, Sensor Networks and Information Processing(ISSNIP),Dec 2011 Adelaide, Australia pp. 401-406.

[14] Rajavara Prasad, P. Rajalakshmi, "Context Aware Building Energy Management System with Heterogeneous Wireless Networks", International Conference on wireless and Mobile Networking Conference,WMNC2013,Dubai,April 2013. 